\title{
THE BOUNDARY VOLUME OF A LATTICE POLYTOPE
}

\author{
GÁBOR HEGEDÜS and ALEXANDER M. KASPRZYK ${ }^{\bowtie}$
}

(Received 3 May 2011)

\begin{abstract}
For a $d$-dimensional convex lattice polytope $P$, a formula for the boundary volume $\operatorname{vol}(\partial P)$ is derived in terms of the number of boundary lattice points on the first $\lfloor d / 2\rfloor$ dilations of $P$. As an application we give a necessary and sufficient condition for a polytope to be reflexive, and derive formulas for the $f$-vector of a smooth polytope in dimensions three, four, and five. We also give applications to reflexive order polytopes, and to the Birkhoff polytope.
\end{abstract}

2010 Mathematics subject classification: primary 52B20; secondary 52C07, 52B12.

Keywords and phrases: lattice polytope, boundary volume, reflexive polytope, order polytope, Birkhoff polytope.

\section{Introduction}

A lattice polytope $P \subset \mathbb{R}^{d}$ is the convex hull of finitely many points in $\mathbb{Z}^{d}$. We shall assume throughout that $P$ is of maximum dimension, so that $\operatorname{dim} P=d$. Denote the boundary of $P$ by $\partial P$. The boundary volume $\operatorname{vol}(\partial P)$ is the volume of each facet of $P$ normalised with respect to the sublattice containing that facet, that is,

$$
\operatorname{vol}(\partial P):=\sum_{F \text { facet of } P} \frac{\operatorname{vol}_{d-1}(F)}{\operatorname{det}\left(\operatorname{aff} F \cap \mathbb{Z}^{d}\right)},
$$

where $\operatorname{vol}_{d-1}(F)$ denotes the $(d-1)$-dimensional volume, and $\operatorname{det}\left(\operatorname{aff} F \cap \mathbb{Z}^{d}\right)$ is the determinant of the sublattice contained in the affine hull of $F$.

In two dimensions, the number of lattice points on the boundary of $P$ is equal to the boundary volume. In three dimensions there is a well-known relationship which can be derived directly from Euler's formula and Pick's theorem (see, for example, [18, Proposition 10.2.3]).

Proposition 1.1. Let P be a three-dimensional convex lattice polytope. Then

$$
\operatorname{vol}(\partial P)=\left|\partial P \cap \mathbb{Z}^{3}\right|-2 .
$$

(C) 2011 Australian Mathematical Publishing Association Inc. 0004-9727/2011 \$16.00 
TABLE 1 . The relationship between the boundary volume and the number of boundary points, for each dimension $4 \leq d \leq 10$ (see Theorem 1.2).

\begin{tabular}{rl}
\hline$d$ & $(d-1) ! \operatorname{vol}(\partial P)$ \\
\hline 4 & $L_{\partial P}(2)-2 L_{\partial P}(1)$ \\
5 & $2\left(L_{\partial P}(2)-4 L_{\partial P}(1)+6\right)$ \\
6 & $L_{\partial P}(3)-4 L_{\partial P}(2)+5 L_{\partial P}(1)$ \\
7 & $2\left(L_{\partial P}(3)-6 L_{\partial P}(2)+15 L_{\partial P}(1)-20\right)$ \\
8 & $L_{\partial P}(4)-6 L_{\partial P}(3)+14 L_{\partial P}(2)-14 L_{\partial P}(1)$ \\
9 & $2\left(L_{\partial P}(4)-8 L_{\partial P}(3)+28 L_{\partial P}(2)-56 L_{\partial P}(1)+70\right)$ \\
10 & $L_{\partial P}(5)-8 L_{\partial P}(4)+27 L_{\partial P}(3)-48 L_{\partial P}(2)+42 L_{\partial P}(1)$ \\
\hline
\end{tabular}

We shall prove the following generalization to arbitrary dimension.

Theorem 1.2. Let P be a d-dimensional convex lattice polytope. Then

$$
\begin{aligned}
\operatorname{vol}(\partial P) & =\frac{\operatorname{det}(\mathcal{A})}{\operatorname{det}(\mathcal{D})} \\
& =\frac{1}{(d-1) !} \sum_{m=0}^{n}(-1)^{n+m}\left(\left(\begin{array}{l}
d-1 \\
n-m
\end{array}\right)+(-1)^{d-1}\left(\begin{array}{l}
d-1 \\
n+m
\end{array}\right)\right)\left|\partial(m P) \cap \mathbb{Z}^{d}\right|
\end{aligned}
$$

where $n:=\lfloor d / 2\rfloor,\left|\partial(0 P) \cap \mathbb{Z}^{d}\right|:=1$, and $\mathcal{A}$ and $\mathcal{D}$ are invertible $n \times n$ matrices defined by

$$
\begin{aligned}
& \mathcal{A}_{i j}:= \begin{cases}\left|\partial(i P) \cap \mathbb{Z}^{d}\right|-2(d-2 n) & \text { if } j=1, \\
i^{d-2 j+1} & \text { otherwise }\end{cases} \\
& \mathcal{D}_{i j}:=i^{d-2 j+1} .
\end{aligned}
$$

The boundary volume formula for each dimension $4 \leq d \leq 10$ is listed in Table 1 .

\section{A general boundary volume formula}

Let $L_{P}(m):=\left|m P \cap \mathbb{Z}^{d}\right|$ denote the number of lattice points in $P$ dilated by a factor of $m \in \mathbb{Z}_{\geq 0}$. Similarly, let $L_{\partial P}(m):=\left|\partial(m P) \cap \mathbb{Z}^{d}\right|$ denote the number of lattice points on the boundary of $m P$. In two dimensions the relationship between $L_{P}$ and $L_{\partial P}$ is given by Pick's theorem [26]. In three dimensions Reeve proved an analogous result.

Theorem 2.1 [28, Theorem 1]. Let $P$ be a three-dimensional convex lattice polytope. Then

$$
2(m-1) m(m+1) \operatorname{vol}(P)=2\left(L_{P}(m)-m\left|P \cap \mathbb{Z}^{3}\right|\right)-\left(L_{\partial P}(m)-m\left|\partial P \cap \mathbb{Z}^{3}\right|\right)
$$

and

$$
L_{\partial P}(m)=2\left(1-m^{2}\right)+m^{2}\left|\partial P \cap \mathbb{Z}^{3}\right|
$$


In general, the function $L_{P}$ is a polynomial of degree $d$, and is called the Ehrhart polynomial. Ehrhart showed that certain coefficients of $L_{P}$ have natural interpretations in terms of $P$.

Theorem 2.2 [13]. Let $P$ be a d-dimensional convex lattice polytope with Ehrhart polynomial $L_{P}(m)=c_{d} m^{d}+\cdots+c_{1} m+c_{0}$. Then:

(i) $c_{d}=\operatorname{vol}(P)$;

(ii) $c_{d-1}=\frac{1}{2} \operatorname{vol}(\partial P)$;

(iii) $c_{0}=1$.

The values of the remaining coefficients of $L_{P}$ have been studied in, for example, [4, 12, 27]. Particular attention has been paid to the connection with toric geometry; under some additional assumptions, the function $L_{P}(m)$ calculates $h^{0}(-m K)$.

Let $P^{\circ}$ denote the strict interior of $P$. Ehrhart conjectured, and Macdonald proved, a remarkable reciprocity formula connecting $L_{P}(m)$ and $L_{P^{\circ}}(m)$ (see [11] for a proof exploiting Serre-Grothendieck duality).

Theorem 2.3 [22]. Let $P$ be a d-dimensional convex lattice polytope. Then

$$
L_{P}(-m)=(-1)^{d} L_{P^{\circ}}(m) .
$$

Since $L_{P}(m)=L_{\partial P}(m)+L_{P^{\circ}}(m)$ we have the following immediate corollary.

Corollary 2.4. Let $P$ be a d-dimensional convex lattice polytope. The coefficients $c_{d-1}, c_{d-3}, c_{d-5}, \ldots$ of $L_{P}$ satisfy the system of equations:

$$
\frac{1}{2} L_{\partial P}(m)=\sum_{i=1}^{\lceil d / 2\rceil} m^{d-2 i+1} c_{d-2 i+1} \quad \text { for all } m \in \mathbb{Z}_{>0} .
$$

A formula for the volume of an even-dimensional convex lattice polytope was derived by Macdonald in [21]:

$$
\operatorname{vol}(P)=\frac{1}{d !}\left(\sum_{m=1}^{d / 2}(-1)^{d / 2-m}\left(\begin{array}{c}
d \\
d / 2-m
\end{array}\right)\left(2\left|(m P)^{\circ} \cap \mathbb{Z}^{d}\right|+\left|\partial(m P) \cap \mathbb{Z}^{d}\right|\right)+(-1)^{d / 2}\left(\begin{array}{c}
d \\
d / 2
\end{array}\right)\right)
$$

Kołodziejczyk was able to compute the odd-dimensional formula in [20]:

$\operatorname{vol}(P)=\frac{1}{(d+1) !} \sum_{m=1}^{(d+1) / 2}(-1)^{(d+1) / 2-m}\left(\begin{array}{c}d+1 \\ (d+1) / 2-m\end{array}\right) m\left(2\left|(m P)^{\circ} \cap \mathbb{Z}^{d}\right|+\left|\partial(m P) \cap \mathbb{Z}^{d}\right|\right)$.

It is worth noticing that, with a little rearranging, one can combine these results to give a general form remarkably similar to (1.2).

Theorem 2.5. Let $P$ be a d-dimensional lattice polytope. Then

$$
\operatorname{vol}(P)=\frac{1}{d !} \sum_{m=0}^{N}(-1)^{N+m}\left(\left(\begin{array}{c}
d \\
N-m
\end{array}\right)+(-1)^{d}\left(\begin{array}{c}
d \\
N+m
\end{array}\right)\right)\left(\left|m P \cap \mathbb{Z}^{d}\right|-\frac{1}{2}\left|\partial(m P) \cap \mathbb{Z}^{d}\right|\right),
$$

where $N:=\lceil d / 2\rceil$ and $\left|\partial(0 P) \cap \mathbb{Z}^{d}\right|:=1$. 
Proof of Theorem 1.2. We wish to express the value of the penultimate coefficient $c_{d-1}$ of $L_{P}$ in terms of $L_{\partial P}$. A formula for $\operatorname{vol}(\partial P)$ follows from Theorem 2.2(ii). We shall handle the even-dimensional and odd-dimensional cases separately. For brevity let us define

$$
b_{m}:=\frac{1}{2 m} L_{\partial P}(m) \quad \text { for all } m \in \mathbb{Z}_{>0} .
$$

When $d=2 n$ is even, Corollary 2.4 tells us that the coefficients satisfy the linear system

$$
\left(\begin{array}{cccc}
1 & 1 & \ldots & 1 \\
1 & 2^{2} & \ldots & 2^{d-2} \\
\vdots & \vdots & & \vdots \\
1 & n^{2} & \ldots & n^{d-2}
\end{array}\right) \cdot\left(\begin{array}{c}
c_{1} \\
c_{3} \\
\vdots \\
c_{d-1}
\end{array}\right)=\left(\begin{array}{c}
b_{1} \\
b_{2} \\
\vdots \\
b_{n}
\end{array}\right)
$$

Equation (1.1) follows from an application of Cramer's rule and some elementary matrix operations.

To obtain the explicit description (1.2), consider the square matrix on the left-hand side of (2.1). This is a Vandermonde matrix; we can express its inverse in terms of the product $U \cdot L$ [34, Equations (5) and (7)], where $U$ is an upper triangular matrix with 1 s on the diagonal, and $L$ is a lower triangular matrix given by

$$
L_{i j}= \begin{cases}0 & \text { if } i<j, \\ 1 & \text { if } i=j=1, \\ \prod_{\substack{k=1 \\ k \neq j} \frac{1}{j^{2}-k^{2}}} & \text { otherwise. }\end{cases}
$$

More explicitly,

$$
\left(\begin{array}{c}
c_{1} \\
c_{3} \\
\vdots \\
c_{d-1}
\end{array}\right)=\left(\begin{array}{cccc}
1 & & \star \\
& 1 & & \\
0 & \ddots & \\
0 & & 1
\end{array}\right) \cdot\left(\begin{array}{cccc}
1 & & & 0 \\
-\frac{1}{3} & \frac{1}{3} & & \\
\vdots & \vdots & \ddots & \\
L_{n 1} & L_{n 2} & \ldots & L_{n n}
\end{array}\right) \cdot\left(\begin{array}{c}
b_{1} \\
b_{2} \\
\vdots \\
b_{n}
\end{array}\right) .
$$

Since we need only know the bottom row of $L$ in order to determine the coefficient $c_{d-1}$, we obtain

$$
\begin{aligned}
c_{d-1} & =\sum_{m=1}^{n}\left(\prod_{\substack{k=1 \\
k \neq m}}^{n} \frac{1}{m^{2}-k^{2}}\right) b_{m} \\
& =2 \sum_{m=1}^{n} \frac{(-1)^{n+m} m^{2}}{(n+m) !(n-m) !} b_{m} \\
& =\frac{1}{(2 n) !} \sum_{m=1}^{n}(-1)^{n+m}\left(\begin{array}{c}
2 n \\
n+m
\end{array}\right) m L_{\partial P}(m) .
\end{aligned}
$$


Observing that

$$
\frac{m}{n}\left(\begin{array}{c}
2 n \\
n+m
\end{array}\right)=\left(\begin{array}{c}
2 n-1 \\
n-m
\end{array}\right)-\left(\begin{array}{c}
2 n-1 \\
n+m
\end{array}\right)
$$

we obtain the result in the even-dimensional case:

$$
c_{d-1}=\frac{1}{2 \cdot(2 n-1) !} \sum_{m=0}^{n}(-1)^{n+m}\left(\left(\begin{array}{c}
2 n-1 \\
n-m
\end{array}\right)-\left(\begin{array}{c}
2 n-1 \\
n+m
\end{array}\right)\right) L_{\partial P}(m) .
$$

When $d=2 n+1$ is odd we obtain the linear system

$$
\left(\begin{array}{cccc}
1 & 1 & \ldots & 1 \\
1 & 2^{2} & \ldots & 2^{d-3} \\
\vdots & \vdots & & \vdots \\
1 & n^{2} & \ldots & n^{d-3}
\end{array}\right) \cdot\left(\begin{array}{c}
c_{2} \\
c_{4} \\
\vdots \\
c_{d-1}
\end{array}\right)=\left(\begin{array}{c}
b_{1}-1 \\
b_{2} / 2-1 / 2^{2} \\
\vdots \\
b_{n} / n-1 / n^{2}
\end{array}\right)
$$

Once again, Cramer's rule yields (1.1).

Solving as in the even case, we have that

$$
c_{d-1}=\frac{1}{(2 n) !} \sum_{m=1}^{n}(-1)^{n+m}\left(\begin{array}{c}
2 n \\
n+m
\end{array}\right)\left(L_{\partial P}(m)-2\right) .
$$

From the identity

$$
\sum_{m=0}^{2 n}(-1)^{m}\left(\begin{array}{l}
2 n \\
m
\end{array}\right)=0
$$

we deduce that

$$
2 \sum_{m=1}^{n}(-1)^{n+m}\left(\begin{array}{c}
2 n \\
n+m
\end{array}\right)=(-1)^{n+1}\left(\begin{array}{c}
2 n \\
n
\end{array}\right)
$$

Hence,

$$
\begin{aligned}
c_{d-1} & =\frac{1}{(2 n) !}\left((-1)^{n}\left(\begin{array}{c}
2 n \\
n
\end{array}\right)+\sum_{m=1}^{n}(-1)^{n+m}\left(\begin{array}{c}
2 n \\
n+m
\end{array}\right) L_{\partial P}(m)\right) \\
& =\frac{1}{2 \cdot(2 n) !}\left(\sum_{m=0}^{n}(-1)^{n+m}\left(\left(\begin{array}{c}
2 n \\
n-m
\end{array}\right)+\left(\begin{array}{c}
2 n \\
n+m
\end{array}\right)\right) L_{\partial P}(m)\right) .
\end{aligned}
$$

This gives us (1.2).

\section{Applications to reflexive polytopes}

In [31] Stanley proved that the generating function for $L_{P}$ can be written as a rational function

$$
\operatorname{Ehr}_{P}(t):=\sum_{m \geq 0} L_{P}(m) t^{m}=\frac{\delta_{0}+\delta_{1} t+\cdots+\delta_{d} t^{d}}{(1-t)^{d+1}}
$$

where the coefficients $\delta_{i}$ are nonnegative. The sequence $\left(\delta_{0}, \delta_{1}, \ldots, \delta_{d}\right)$ is known as the $\delta$-vector of $P$. For an elementary proof of this and other relevant results, see $[6,7]$. 
The following corollary is a consequence of Theorem 2.2.

Corollary 3.1. Let $P$ be a d-dimensional convex lattice polytope with $\delta$-vector $\left(\delta_{0}, \delta_{1}, \ldots, \delta_{d}\right)$. Then:

(i) $\delta_{0}=1$;

(ii) $\delta_{1}=\left|P \cap \mathbb{Z}^{d}\right|-d-1$;

(iii) $\delta_{d}=\left|P^{\circ} \cap \mathbb{Z}^{d}\right|$;

(iv) $\delta_{0}+\cdots+\delta_{d}=d ! \operatorname{vol}(P)$.

Hibi proved [17] the following lower bound on the $\delta_{i}$, commonly referred to as the lower bound theorem.

Theorem 3.2. Let $P$ be a d-dimensional convex lattice polytope with $\left|P^{\circ} \cap \mathbb{Z}^{d}\right|>0$. Then $\delta_{1} \leq \delta_{i}$ for every $2 \leq i \leq d-1$.

As a consequence of the lower bound theorem we have a bound on the number of lattice points in $P$ in terms of the volume of $P$. Note that this bound is sharp: equality is given in each dimension by the $d$-simplex $\operatorname{conv}\left\{e_{1}, \ldots, e_{d},-e_{1}-\cdots-e_{d}\right\}$, where $e_{1}, \ldots, e_{d}$ is a basis of $\mathbb{Z}^{d}$.

Corollary 3.3. Let $P$ be a d-dimensional convex lattice polytope with $\left|P^{\circ} \cap \mathbb{Z}^{d}\right|>0$. Then

$$
d ! \operatorname{vol}(P) \geq(d-1)\left|P \cap \mathbb{Z}^{d}\right|-d^{2}+3 .
$$

We have equality if and only if the $\delta$-vector of $P$ equals

$$
\left(1,\left|P \cap \mathbb{Z}^{d}\right|-d-1,\left|P \cap \mathbb{Z}^{d}\right|-d-1, \ldots,\left|P \cap \mathbb{Z}^{d}\right|-d-1,1\right) .
$$

Proof. This is a consequence of Corollary 3.1 parts (ii) and (iv), and Theorem 3.2.

A convex lattice polytope $P$ is called Fano if $P^{\circ} \cap \mathbb{Z}^{d}=\{0\}$; that is, if the origin is the only interior lattice point of $P$. A convex lattice polytope $P$ is called reflexive if the dual polyhedron

$$
P^{\vee}:=\left\{u \in \mathbb{R}^{d} \mid\langle u, v\rangle \leq 1 \text { for all } v \in P\right\}
$$

is also a lattice polytope. Clearly any reflexive polytope is Fano. Reflexive polytopes are of particular relevance to toric geometry: they correspond to Gorenstein toric Fano varieties (see [2]). There are many interesting characterizations of reflexive polytopes (for example, the list in [15]).

Theorem 3.4. Let $P$ be a d-dimensional Fano polytope. The following are equivalent:

(i) $P$ is reflexive;

(ii) $L_{P}(m)=L_{\partial P}(m)+L_{P}(m-1)$ for all $m \in \mathbb{Z}_{>0}$;

(iii) $d \operatorname{vol}(P)=\operatorname{vol}(\partial P)$;

(iv) $\delta_{i}=\delta_{d-i}$ for all $0 \leq i \leq d$.

Theorem 3.4(iv) is commonly known as Hibi's palindromic theorem [16] and can be generalized to duals of nonreflexive polytopes [14]. It is a consequence of a more 
general result of Stanley's [30] concerning Gorenstein rings. Clearly any polytope giving equality in Corollary 3.3 must be reflexive.

REMARK 3.5. Of course, as a consequence of (1.2) and Theorem 3.4(iii), one can add the equivalent condition:

$$
\operatorname{vol}(P)=\frac{1}{d !} \sum_{m=0}^{n}(-1)^{n+m}\left(\left(\begin{array}{l}
d-1 \\
n-m
\end{array}\right)+(-1)^{d-1}\left(\begin{array}{l}
d-1 \\
n+m
\end{array}\right)\right)\left|\partial(m P) \cap \mathbb{Z}^{d}\right| .
$$

We are now in a position to express the volume of a reflexive polytope in terms of the number of lattice points in the first $n$ dilations.

Corollary 3.6. Let $P$ be a d-dimensional reflexive polytope. Then

$$
\operatorname{vol}(P)=\frac{1}{d !} \sum_{m=0}^{n}(-1)^{n+m}\left(\left(\begin{array}{c}
d \\
n-m
\end{array}\right)+(-1)^{d-1}\left(\begin{array}{c}
d \\
n+m+1
\end{array}\right)\right)\left|m P \cap \mathbb{Z}^{d}\right|
$$

where $n:=\lfloor d / 2\rfloor$.

Proof. This follows from Theorem 3.4(ii), Remark 3.5, and the recursive definition of the binomial coefficient.

It is tempting to conjecture that the converse of Corollary 3.6 is true. However, suppose that $P$ is a three-dimensional convex lattice polytope satisfying (3.1). By Theorem 2.1,

$$
L_{P}(m)-L_{\partial P}(m)-L_{P}(m-1)=\left(\left|P \cap \mathbb{Z}^{3}\right|-\left|\partial P \cap \mathbb{Z}^{3}\right|-1\right)\left(m^{2}-m+1\right) .
$$

Thus we require the additional assumption that $\left|P^{\circ} \cap \mathbb{Z}^{3}\right|=1$; only then would it follow (by Theorem 3.4(ii)) that $P$ is reflexive.

More generally we can make use of Theorems 1.2 and 2.5 to write down a necessary and sufficient relation between the number of points in, and on the boundary of, the first $N$ dilations of $P$.

Theorem 3.7. Let $P$ be a d-dimensional Fano polytope. Then $P$ is reflexive if and only if

$$
0= \begin{cases}\sum_{m=0}^{N}(-1)^{N+m}\left(\begin{array}{c}
2 N \\
N+m
\end{array}\right) & \\
\times\left(d\left|m P \cap \mathbb{Z}^{d}\right|-(N+m)\left|\partial(m P) \cap \mathbb{Z}^{d}\right|\right) & \text { if d is even, } \\
\sum_{m=0}^{N}(-1)^{N+m}\left(\begin{array}{c}
2 N \\
N+m
\end{array}\right) & \\
\times\left(m d\left|m P \cap \mathbb{Z}^{d}\right|+\left(N^{2}-m^{2}-\frac{m d}{2}\right)\left|\partial(m P) \cap \mathbb{Z}^{d}\right|\right) & \text { if } d \text { is odd, }\end{cases}
$$

where $N:=\lceil d / 2\rceil$ and $\left|\partial(0 P) \cap \mathbb{Z}^{d}\right|:=1$. 
Proof. Suppose first that $d$ is even, so that $N=n$. By Theorem 3.4(iii), $P$ is reflexive if and only if

$$
\begin{aligned}
& \sum_{m=0}^{n}(-1)^{n+m}\left(\left(\begin{array}{l}
d-1 \\
n-m
\end{array}\right)-\left(\begin{array}{l}
d-1 \\
n+m
\end{array}\right)\right)\left|\partial(m P) \cap \mathbb{Z}^{d}\right| \\
& \quad=\sum_{m=0}^{n}(-1)^{n+m}\left(\left(\begin{array}{c}
d \\
n-m
\end{array}\right)+\left(\begin{array}{c}
d \\
n+m
\end{array}\right)\right)\left(\left|m P \cap \mathbb{Z}^{d}\right|-\frac{1}{2}\left|\partial(m P) \cap \mathbb{Z}^{d}\right|\right),
\end{aligned}
$$

where the left-hand side follows from Theorem 1.2, and the right-hand side from Theorem 2.5.

Using the binomial identity

$$
\left(\begin{array}{l}
d-1 \\
n-m
\end{array}\right)-\left(\begin{array}{l}
d-1 \\
n+m
\end{array}\right)=\frac{2 m}{d}\left(\begin{array}{c}
d \\
n+m
\end{array}\right)
$$

we have that

$$
\begin{aligned}
& \sum_{m=0}^{n}(-1)^{n+m}\left(\begin{array}{c}
d \\
n+m
\end{array}\right) m\left|\partial(m P) \cap \mathbb{Z}^{d}\right| \\
& \quad=d \sum_{m=0}^{n}(-1)^{n+m}\left(\begin{array}{c}
d \\
n+m
\end{array}\right)\left(\left|m P \cap \mathbb{Z}^{d}\right|-\frac{1}{2}\left|\partial(m P) \cap \mathbb{Z}^{d}\right|\right) .
\end{aligned}
$$

Noticing that $d / 2=n$, we obtain our result.

Now suppose that $d$ is odd. In particular, $N=n+1$. In this case we have that $P$ is reflexive if and only if

$$
\begin{aligned}
& \sum_{m=0}^{n}(-1)^{n+m} 2\left(\begin{array}{l}
d-1 \\
n+m
\end{array}\right)\left|\partial(m P) \cap \mathbb{Z}^{d}\right| \\
& \quad=\sum_{m=0}^{n+1}(-1)^{n+m}\left(\left(\begin{array}{c}
d \\
n+m+1
\end{array}\right)-\left(\begin{array}{c}
d \\
n+m
\end{array}\right)\right)\left(\left|m P \cap \mathbb{Z}^{d}\right|-\frac{1}{2}\left|\partial(m P) \cap \mathbb{Z}^{d}\right|\right) .
\end{aligned}
$$

By standard binomial identities,

$$
\begin{aligned}
\left(\begin{array}{l}
d-1 \\
n+m
\end{array}\right) & =\frac{n+m+1}{d}\left(\begin{array}{c}
d \\
n+m+1
\end{array}\right) \\
& =\frac{n+m+1}{d}\left(\begin{array}{c}
d \\
n-m
\end{array}\right) \\
& =\frac{(n+m+1)(n-m+1)}{d(d+1)}\left(\begin{array}{c}
d+1 \\
n+m+1
\end{array}\right)
\end{aligned}
$$

and

$$
\left(\begin{array}{c}
d \\
n+m+1
\end{array}\right)-\left(\begin{array}{c}
d \\
n-m+1
\end{array}\right)=-\frac{2 m}{d+1}\left(\begin{array}{c}
d+1 \\
n+m+1
\end{array}\right)
$$


TABle 2. A $d$-dimensional Fano polytope $P$ is reflexive if and only if the expression $f(P)$ in the second column vanishes (see Theorem 3.7).

\begin{tabular}{ll}
\hline$d$ & $f(P)$ \\
\hline 3 & $2 L_{P}(2)-L_{\partial P}(2)-4 L_{P}(1)-2 L_{\partial P}(1)+8$ \\
4 & $L_{P}(2)-L_{\partial P}(2)-4 L_{P}(1)+3 L_{\partial P}(1)+3$ \\
5 & $2 L_{P}(3)-L_{\partial P}(3)-8 L_{P}(2)+10 L_{P}(1)+11 L_{\partial P}(1)-24$ \\
6 & $L_{P}(3)-L_{\partial P}(3)-6 L_{P}(2)+5 L_{\partial P}(2)+15 L_{P}(1)-10 L_{\partial P}(1)-10$ \\
7 & $2 L_{P}(4)-L_{\partial P}(4)-12 L_{P}(3)+2 L_{\partial P}(3)+28 L_{P}(2)$ \\
& $\quad+10 L_{\partial P}(2)-28 L_{P}(1)-46 L_{\partial P}(1)+80$ \\
8 & $L_{P}(4)-L_{\partial P}(4)-8 L_{P}(3)+7 L_{\partial P}(3)+28 L_{P}(2)-21 L_{\partial P}(2)$ \\
& $\quad-56 L_{P}(1)+35 L_{\partial P}(1)+35$ \\
\hline
\end{tabular}

Observing that $n-m+1$ vanishes when $m=n+1$, we obtain the equality

$$
\begin{aligned}
& \sum_{m=0}^{n+1}(-1)^{n+m}(n+m+1)(n-m+1)\left(\begin{array}{c}
d+1 \\
n+m+1
\end{array}\right)\left|\partial(m P) \cap \mathbb{Z}^{d}\right| \\
& =\sum_{m=0}^{n+1}(-1)^{n+m+1} m d\left(\begin{array}{c}
d+1 \\
n+m+1
\end{array}\right)\left(\left|m P \cap \mathbb{Z}^{d}\right|-\frac{1}{2}\left|\partial(m P) \cap \mathbb{Z}^{d}\right|\right)
\end{aligned}
$$

which is equivalent to

$$
\sum_{m=0}^{N}(-1)^{N+m}\left(\begin{array}{c}
d+1 \\
N+m
\end{array}\right)\left(m d\left|m P \cap \mathbb{Z}^{d}\right|+\left((N+m)(N-m)-\frac{m d}{2}\right)\left|\partial(m P) \cap \mathbb{Z}^{d}\right|\right)=0
$$

The conditions given by Theorem 3.7 are summarized in Table 2 for low dimensions.

Notice that if $P$ is a reflexive polytope and $d$ is even then, by Theorem 3.4(ii), Theorem 3.7 reduces to

$$
\begin{aligned}
& 0=\sum_{m=0}^{n}(-1)^{n+m}\left(\begin{array}{c}
d \\
n+m
\end{array}\right)\left(d\left|m P \cap \mathbb{Z}^{d}\right|-(n+m)\left(\left|m P \cap \mathbb{Z}^{d}\right|-\left|(m-1) P \cap \mathbb{Z}^{d}\right|\right)\right) \\
& =\sum_{m=0}^{n-1}(-1)^{n+m}\left(\begin{array}{c}
2 n \\
n-m
\end{array}\right)(n-m)\left|m P \cap \mathbb{Z}^{d}\right| \\
& -\sum_{m=0}^{n-1}(-1)^{n+m}\left(\begin{array}{c}
2 n \\
n+m+1
\end{array}\right)(n+m+1)\left|m P \cap \mathbb{Z}^{d}\right| .
\end{aligned}
$$

Clearly the right-hand side vanishes, so we learn nothing new. The odd-dimensional case is different; the relation is given in Theorem 3.8 and calculated for small dimensions in Table 3. 
TABLe 3. If $P$ is a $d$-dimensional reflexive polytope then the expression $g(P)$ in the second column will vanish (see Theorem 3.8).

\begin{tabular}{ll}
\hline$d$ & $g(P)$ \\
\hline 3 & $L_{P}(2)-5 L_{P}(1)+10$ \\
5 & $L_{P}(3)-7 L_{P}(2)+21 L_{P}(1)-35$ \\
7 & $L_{P}(4)-9 L_{P}(3)+36 L_{P}(2)-84 L_{P}(1)+126$ \\
9 & $L_{P}(5)-11 L_{P}(4)+55 L_{P}(3)-165 L_{P}(2)+330 L_{P}(1)-462$ \\
\hline
\end{tabular}

Theorem 3.8. Let $P$ be a reflexive d-dimensional polytope, where $d$ is odd. Then

$$
\sum_{m=0}^{N}(-1)^{N+m}\left(\begin{array}{c}
d+2 \\
N-m
\end{array}\right)\left|m P \cap \mathbb{Z}^{d}\right|=0,
$$

where $N:=\lceil d / 2\rceil$.

Proof. From Theorems 3.4(ii) and 3.7

$$
\begin{aligned}
0=(-1)^{N}\left(\begin{array}{c}
2 N \\
N
\end{array}\right) N^{2}+\sum_{m=1}^{N}(-1)^{N+m}\left(\begin{array}{c}
2 N \\
N+m
\end{array}\right) \\
\quad \times\left(m d\left|m P \cap \mathbb{Z}^{d}\right|+\left(N^{2}-m^{2}-\frac{m d}{2}\right)\left(\left|m P \cap \mathbb{Z}^{d}\right|-\left|(m-1) P \cap \mathbb{Z}^{d}\right|\right)\right) \\
=\sum_{m=0}^{N}(-1)^{N+m}\left(\begin{array}{c}
2 N \\
N+m
\end{array}\right)\left(\frac{m d}{2}+N^{2}-m^{2}\right)\left|m P \cap \mathbb{Z}^{d}\right| \\
\quad-\sum_{m=0}^{N}(-1)^{N+m}\left(\begin{array}{c}
2 N \\
N+m+1
\end{array}\right)\left(\frac{(m+1) d}{2}-N^{2}+(m+1)^{2}\right)\left|m P \cap \mathbb{Z}^{d}\right| .
\end{aligned}
$$

Now

$$
\begin{aligned}
\left(\begin{array}{c}
2 N \\
N+m
\end{array}\right)\left(\frac{m d}{2}+N^{2}-m^{2}\right)-\left(\begin{array}{c}
2 N \\
N+M+1
\end{array}\right)\left(\frac{(m+1) d}{2}-N^{2}+(m+1)^{2}\right) \\
=\left(\left(\begin{array}{c}
2 N \\
N+m
\end{array}\right)-\left(\begin{array}{c}
2 N \\
N+m+1
\end{array}\right)\right) \frac{m d}{2}+\left(\left(\begin{array}{c}
2 N \\
N+m
\end{array}\right)+\left(\begin{array}{c}
2 N \\
N+m+1
\end{array}\right)\right)\left(N^{2}-m^{2}\right) \\
\\
\quad-\left(\begin{array}{c}
2 N \\
N+m+1
\end{array}\right)\left(2 m+1+\frac{d}{2}\right),
\end{aligned}
$$

which, by standard results on the binomial coefficient, reduces to

$$
\begin{gathered}
\left(\begin{array}{c}
2 N+1 \\
N-m
\end{array}\right) \frac{m d(2 m+1)}{2(2 N+1)}+\left(\begin{array}{c}
2 N+1 \\
N-m
\end{array}\right)\left(N^{2}-m^{2}\right)-\left(\begin{array}{c}
2 N+1 \\
N-m
\end{array}\right)\left(2 m+1+\frac{d}{2}\right) \frac{N-m}{2 N+1} \\
\quad=\left(\begin{array}{c}
2 N+1 \\
N-m
\end{array}\right) \frac{1}{2 N+1}\left(\frac{m d}{2}(2 m+1)+(N-m)\left(2 N^{2}+N+2 m N-m-\frac{d}{2}-1\right)\right) .
\end{gathered}
$$


Since $d=2 N-1$ we can simplify the term in brackets:

$$
\begin{aligned}
& \frac{m d}{2}(2 m+1)+(N-m)\left(2 N^{2}+N+2 m N-m-\frac{d}{2}-1\right) \\
& =\frac{m d}{2}(2 m+1)+(N-m)\left(2 N^{2}+N+m d-\frac{d}{2}-1\right) \\
& =\frac{m d}{2}(2 N+1)+(N-m)\left(2 N^{2}-\frac{1}{2}\right) \\
& \quad=\frac{N(2 N-1)(2 N+1)}{2} .
\end{aligned}
$$

Thus

$$
\frac{N(2 N-1)}{2} \sum_{m=0}^{N}(-1)^{N+m}\left(\begin{array}{c}
2 N+1 \\
N-m
\end{array}\right)\left|m P \cap \mathbb{Z}^{d}\right|=0 .
$$

Finally, since we are free to divide through by a nonzero constant, we obtain our result.

By exploiting Hibi's palindromic theorem one can express the $\delta_{i}$ in terms of $L_{P}(m)$, for $1 \leq m \leq\lfloor d / 2\rfloor$. When $d=4$ we obtain the $\delta$-vector

$$
\left(1,\left|P \cap \mathbb{Z}^{4}\right|-5,\left|2 P \cap \mathbb{Z}^{4}\right|-5\left|P \cap \mathbb{Z}^{4}\right|+10,\left|P \cap \mathbb{Z}^{4}\right|-5,1\right),
$$

and when $d=5$ we have

$$
\begin{aligned}
& \left(1,\left|P \cap \mathbb{Z}^{5}\right|-6,\left|2 P \cap \mathbb{Z}^{5}\right|-6\left|P \cap \mathbb{Z}^{5}\right|+15,\right. \\
& \left.\quad\left|2 P \cap \mathbb{Z}^{5}\right|-6\left|P \cap \mathbb{Z}^{5}\right|+15,\left|P \cap \mathbb{Z}^{5}\right|-6,1\right) .
\end{aligned}
$$

Corollary 3.9. If $P$ is a four-dimensional reflexive polytope then the following bound is sharp:

$$
6\left|P \cap \mathbb{Z}^{4}\right| \leq\left|2 P \cap \mathbb{Z}^{4}\right|+15
$$

If $P$ is a five-dimensional reflexive polytope then the following bounds are sharp:

$$
\begin{aligned}
& \left|P \cap \mathbb{Z}^{5}\right| \leq \frac{1}{7}\left|2 P \cap \mathbb{Z}^{5}\right|+3, \\
& \left|2 P \cap \mathbb{Z}^{5}\right| \leq \frac{1}{4}\left|3 P \cap \mathbb{Z}^{5}\right|+7 .
\end{aligned}
$$

Proof. By Theorem 3.2, $\delta_{1} \leq \delta_{2}$. Applying this to (3.2) gives

$$
\begin{aligned}
& 6\left|P \cap \mathbb{Z}^{4}\right| \leq\left|2 P \cap \mathbb{Z}^{4}\right|+15 \quad \text { when } d=4, \\
& \left|P \cap \mathbb{Z}^{5}\right| \leq \frac{1}{7}\left|2 P \cap \mathbb{Z}^{5}\right|+3 \quad \text { when } d=5 .
\end{aligned}
$$

In the case where $d=5$ we apply Theorem 3.8 to (3.3), obtaining the second bound.

If $P$ is a four-dimensional reflexive polytope such that $6\left|P \cap \mathbb{Z}^{4}\right|=\left|2 P \cap \mathbb{Z}^{4}\right|+15$ then it has $\delta$-vector

$$
\left(1,\left|P \cap \mathbb{Z}^{4}\right|-5,\left|P \cap \mathbb{Z}^{4}\right|-5,\left|P \cap \mathbb{Z}^{4}\right|-5,1\right)
$$

and $4 ! \operatorname{vol}(P)=3\left|P \cap \mathbb{Z}^{4}\right|-13$. These conditions are satisfied by the simplex associated with $\mathbb{P}^{4}$ (see the remark preceding Corollary 3.3). 
TABLE 4. The smooth polytopes attaining the bounds in Corollary 3.9. The ID refers to the ID of the polytope in the online Graded Ring Database; the data was calculated using [23].

\begin{tabular}{ll}
\hline$d$ & ID \\
\hline 4 & $24,25,127,128,138,139,144,145,147$ \\
5 & $148,149,950,954,955,989,990,1008,1009,1010,1013$ \\
\hline
\end{tabular}

Suppose that $P$ is a five-dimensional reflexive polytope attaining both of the bounds above. Then

$$
\left|2 P \cap \mathbb{Z}^{5}\right|=7\left|P \cap \mathbb{Z}^{5}\right|-21
$$

and

$$
\left|3 P \cap \mathbb{Z}^{5}\right|=28\left|P \cap \mathbb{Z}^{5}\right|-112 .
$$

In particular, the $\delta$-vector is given by

$$
\left(1,\left|P \cap \mathbb{Z}^{5}\right|-6,\left|P \cap \mathbb{Z}^{5}\right|-6,\left|P \cap \mathbb{Z}^{5}\right|-6,\left|P \cap \mathbb{Z}^{5}\right|-6,1\right),
$$

and 5 ! $\operatorname{vol}(P)=4\left|P \cap \mathbb{Z}^{5}\right|-22$. An example satisfying these conditions is the simplex associated with $\mathbb{P}^{5}$.

The examples given in Corollary 3.9 are not unique. A search through Øbro's classification of the smooth polytopes in dimensions four and five (which form a subset of the reflexive polytopes) gives many more examples. ${ }^{1}$ These are recorded in Table 4.

\section{Applications to smooth polytopes}

Let the number of $i$-dimensional faces of a polytope $P$ be denoted by $f_{i}$. The vector $\left(f_{0}, f_{1}, \ldots, f_{d-1}\right)$ is called the $f$-vector of $P$. By convention $f_{-1}=f_{d}=1$, representing the empty face $\emptyset$ and the entire polytope $P$. The $f$-vector satisfies Euler's relation

$$
\sum_{i=-1}^{d}(-1)^{i} f_{i}=0 .
$$

When $P$ is simplicial (that is, the facets of $P$ are $(d-1)$-simplices) the DehnSommerville equations give some additional relations amongst the $f_{i}$. Conjectured by Dehn and first proved by Sommerville, these equations did not become widely known until they were rediscovered by Klee.

Theorem 4.1 [19]. Let P be a d-dimensional simplicial lattice polytope with $f$-vector $\left(f_{0}, f_{1}, \ldots, f_{d-1}\right)$. Then

$$
f_{i}=\sum_{j=i}^{d-1}(-1)^{d-1-j}\left(\begin{array}{l}
j+1 \\
i+1
\end{array}\right) f_{j} \quad \text { for } 1 \leq i \leq d-2 .
$$

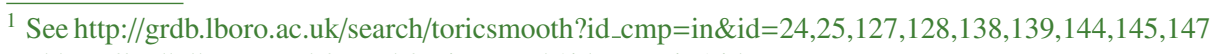
and http://grdb.lboro.ac.uk/search/toricsmooth?id_cmp=in\&id= $148,149,950,954,955,989,990,1008,1009,1010,1013$.
} 
A $d$-dimensional convex lattice polytope $P$ is called smooth if the vertices of any facet of $P$ form a $\mathbb{Z}$-basis of the ambient lattice $\mathbb{Z}^{d}$. Any such $P$ is simplicial and reflexive. Smooth polytopes are in bijective correspondence with smooth toric Fano varieties, and as such have been the subject of much study (see, for example, [1,23]).

In [25] Park investigated the $f$-vector of smooth polytopes of dimension $3 \leq d \leq 5$ and established weak bounds on the $f_{i}$. We shall make use of Theorem 1.2 to give an explicit description of the $f$-vector in those dimensions.

THEOREM 4.2. If $P$ is a three-dimensional smooth polytope then its $f$-vector is given by

$$
\left(\left|\partial P \cap \mathbb{Z}^{3}\right|, 3\left|\partial P \cap \mathbb{Z}^{3}\right|-6,2\left|\partial P \cap \mathbb{Z}^{3}\right|-4\right) .
$$

If $P$ is a four-dimensional smooth polytope then its $f$-vector is given by

$$
\begin{aligned}
& \left(\left|\partial P \cap \mathbb{Z}^{4}\right|,\left|\partial(2 P) \cap \mathbb{Z}^{4}\right|-\left|\partial P \cap \mathbb{Z}^{4}\right|, 2\left|\partial(2 P) \cap \mathbb{Z}^{4}\right|-4\left|\partial P \cap \mathbb{Z}^{4}\right|,\right. \\
& \left.\left|\partial(2 P) \cap \mathbb{Z}^{4}\right|-2\left|\partial P \cap \mathbb{Z}^{4}\right|\right) .
\end{aligned}
$$

If $P$ is a five-dimensional smooth polytope then its $f$-vector is given by

$$
\begin{array}{r}
\left(\left|\partial P \cap \mathbb{Z}^{5}\right|,\left|\partial(2 P) \cap \mathbb{Z}^{5}\right|-\left|\partial P \cap \mathbb{Z}^{5}\right|, 4\left|\partial(2 P) \cap \mathbb{Z}^{5}\right|-14\left|\partial P \cap \mathbb{Z}^{5}\right|+20\right. \\
\left.5\left|\partial(2 P) \cap \mathbb{Z}^{5}\right|-20\left|\partial P \cap \mathbb{Z}^{5}\right|+30,2\left|\partial(2 P) \cap \mathbb{Z}^{5}\right|-8\left|\partial P \cap \mathbb{Z}^{5}\right|+12\right) .
\end{array}
$$

Proof. Let $P$ be a $d$-dimensional smooth polytope. By definition each facet $F$ of $P$ is a simplex whose vertices generate the underlying lattice $\mathbb{Z}^{d}$. Hence $\operatorname{vol}(F)=1 /(d-1)$ !, so

$$
(d-1) ! \operatorname{vol}(\partial P)=f_{d-1}
$$

Furthermore, $\left|\partial P \cap \mathbb{Z}^{n}\right|=f_{0}$.

$d=3$ : Theorem 4.1 gives $2 f_{1}=3 f_{2}$, and Theorem 1.2 yields $f_{2}=2 f_{0}-4$. Thus the $f$-vector is uniquely defined in terms of $f_{0}$.

$d=4$ : In this case Theorem 4.1 gives $f_{2}=2 f_{3}$. Applying (4.1) we obtain $f_{1}=f_{0}+f_{3}$. Finally, Theorem 1.2 tells us that $f_{3}=\left|\partial(2 P) \cap \mathbb{Z}^{4}\right|-2 f_{0}$. The result follows. $d=5$ : In dimension five Theorem 4.1 and (4.1) give three relations:

$$
\begin{aligned}
2 f_{1} & =3 f_{2}-5 f_{4}, \\
2 f_{3} & =5 f_{4}, \\
2 f_{0}-f_{2}+2 f_{4} & =4 .
\end{aligned}
$$

From Theorem 1.2 we know that $f_{4}=2\left|\partial(2 P) \cap \mathbb{Z}^{5}\right|-8 f_{0}+12$. Substituting, we see that the $f$-vector is uniquely defined in terms of $\left|\partial(2 P) \cap \mathbb{Z}^{5}\right|$ and $\left|\partial P \cap \mathbb{Z}^{5}\right|$.

It is worth noting that Casagrande [10] proves a sharp bound for $\left|\partial P \cap \mathbb{Z}^{d}\right|$ in terms of the dimension, and Batyrev [3, Theorem 2.3.7] gives us a bound on $f_{d-3}$ in terms of $f_{d-2}$. Bremner and Klee [8] provide a lower bound on $f_{1}$ in terms of $f_{0}$ and $d$. These results are collected in the following theorem. 
Theorem 4.3. Let $P$ be d-dimensional smooth polytope. Then the following inequalities hold:

(i) $\left|\partial P \cap \mathbb{Z}^{d}\right| \leq \begin{cases}3 d & \text { if } d \text { is even; } \\ 3 d-1 & \text { if } d \text { is odd. }\end{cases}$

(ii) $12 f_{d-3} \geq(3 d-4) f_{d-2}$.

(iii) $d f_{0} \leq f_{1}+\left(\begin{array}{c}d+1 \\ 2\end{array}\right)$.

Thus we obtain upper and lower bounds on $\left|\partial(2 P) \cap \mathbb{Z}^{d}\right|$ when $d=4$ or 5 .

COROLlary 4.4. If $P$ is a four-dimensional smooth polytope then

$$
5\left|\partial P \cap \mathbb{Z}^{4}\right|-10 \leq\left|\partial(2 P) \cap \mathbb{Z}^{4}\right| \leq 5\left|\partial P \cap \mathbb{Z}^{4}\right| .
$$

If $P$ is a five-dimensional smooth polytope then

$$
42\left|\partial P \cap \mathbb{Z}^{5}\right|-105 \leq 7\left|\partial(2 P) \cap \mathbb{Z}^{5}\right| \leq 52\left|\partial P \cap \mathbb{Z}^{5}\right|-90 .
$$

Proor. Apply Theorem 4.3(ii) and (iii) to Theorem 4.2.

COROLlary 4.5. If $P$ is a four-dimensional smooth polytope then

$$
4 ! \operatorname{vol}(P) \leq 3 f_{0} .
$$

If $P$ is a five-dimensional smooth polytope then

$$
5 ! \operatorname{vol}(P) \leq \frac{48 f_{0}-96}{7} .
$$

Proof. Recall that since $P$ is smooth, $d ! \operatorname{vol}(P)=(d-1) ! \operatorname{vol}(\partial P)=f_{d-1}$. In each case Theorem 4.2 tells us the value for $f_{d-1}$. Applying Corollary 4.5 immediately gives the result in dimension four.

In dimension five we see that

$$
\begin{aligned}
7 \cdot 5 ! \operatorname{vol}(P) & =7 \cdot 4 ! \operatorname{vol}(\partial P) \\
& =2\left(7\left|\partial(2 P) \cap \mathbb{Z}^{5}\right|-28\left|\partial P \cap \mathbb{Z}^{5}\right|+42\right) \\
& \leq 2\left(24 f_{0}-48\right),
\end{aligned}
$$

where the final inequality is an application of Corollary 4.5.

The smooth polytopes attaining either the lower or the upper limit in Corollary 4.4 are listed in Table 5. ${ }^{1}$ The upper bound in dimension five is not sharp.

\section{Reflexive order polytopes}

Let $Q$ be a finite poset with $d:=|Q|$ elements. Let $\Omega(Q, k)$ denote the number of order-preserving maps $f: Q \rightarrow C_{k}$, where $C_{k}$ is the chain with $k \in \mathbb{Z}_{>0}$ elements; that is, if $x \leq y$ in $Q$, then $f(x) \leq f(y)$. Then $\Omega(Q, k)$ is a polynomial in $k$ of degree $d$, called the order polynomial of $Q$.

\footnotetext{
${ }^{1}$ See http://grdb.lboro.ac.uk/search/toricsmooth?id_cmp=in\&id=24,25,127,128,138,139,144,145,147 and http://grdb.lboro.ac.uk/search/toricsmooth?id_cmp=in\&id= $148,149,950,954,955,989,990,1008,1009,1010,1013$.
} 
TABLE 5. The smooth polytopes attaining one of the bounds in Corollary 4.4. The ID refers to the ID of the polytope in the online Graded Ring Database; the data was calculated using [23].

\begin{tabular}{lll}
\hline$d$ & Equality & ID \\
\hline \multirow{2}{*}{4} & Lower & $24,25,127,128,138,139,144,145,147$ \\
& Upper & 63,100 \\
& Lower & $148,149,950,954,955,989,990,1008,1009,1010,1013$ \\
& Upper & None \\
\hline
\end{tabular}

Let $\bar{\Omega}(Q, k)$ denote the number of strictly order-preserving maps $f: Q \rightarrow C_{k}$; that is, if $x<y$ in $Q$, then $f(x)<f(y)$. Once again $\bar{\Omega}(Q, k)$ is a polynomial in $k$ of degree $d$; it is called the strict order polynomial of $Q$.

Definition 5.1. A poset $Q$ is said to be graded if there exists an order-preserving function $f$ such that whenever $y$ covers $x, f(y)=f(x)+1$. Equivalently, all maximal chains of $Q$ have the same length $r$. Following Stanley [33, Ch. 3.1] we shall call $r$ the rank of $Q$. In particular, one can adjoin a unique minimum element $\hat{0}$ and unique maximum element $\hat{1}$ to $Q$ to obtain a bounded, graded poset $\hat{Q}$ of rank $r+2$.

Definition 5.2. A bijective order-preserving map is called a linear extension of $Q$. The number of linear extensions is denoted by $e(Q)$.

Let $e_{s}(Q)$ denote the number of surjective order-preserving maps $f: Q \rightarrow C_{s}$.

ExAmple 5.3. If $Q$ is the antichain with $|Q|=d$ then we have $\bar{\Omega}(Q, k)=\Omega(Q, k)=k^{d}$ and $e(Q)=d$ !. If $Q$ is the chain $C_{d}$ then

$$
\Omega(Q, k)=\left(\begin{array}{c}
d+k-1 \\
d
\end{array}\right), \quad \bar{\Omega}(Q, k)=\left(\begin{array}{l}
k \\
d
\end{array}\right) \quad \text { and } \quad e(Q)=1 .
$$

THEOREM 5.4 [29]. Let $Q$ be a finite poset with $|Q|=d$ and order polynomial $\Omega(Q, k)=$ $a_{d} k^{d}+\cdots+a_{1} k+a_{0}$. Then:

(i) $\bar{\Omega}(Q, k)=(-1)^{d} \Omega(Q,-k)$ for all $k \in \mathbb{Z}$;

(ii) if $Q$ is graded of rank $r$, then $a_{d-1}=r e(Q) / 2(d-1)$ !;

(iii) if $Q$ is graded of rank $r$, then $\Omega(Q,-r-k)=(-1)^{d} \Omega(Q, k)$ for each $k \in \mathbb{Z}$;

(iv) $a_{d}=e(Q) / d$ !;

(v) $\Omega(Q, k)=\sum_{s=1}^{d} e_{s}(Q)\left(\begin{array}{l}k \\ s\end{array}\right)$;

(vi) if $Q$ is graded of rank $r$, then $2 e_{d-1}(Q)=(d-r+1) e(Q)$.

Theorem 5.4(i) is commonly referred to as the reciprocity theorem for the order polynomial.

Definition 5.5. The order polytope $O(Q)$ of a poset $Q$ is the set of order-preserving maps from $Q$ to the interval $[0,1]$, that is, the set of all functions $f$ satisfying

$$
\begin{array}{ll}
0 \leq f(x) \leq 1 & \text { for all } x \in Q \\
f(x) \leq f(y) & \text { if } y \text { covers } x \text { in Q. }
\end{array}
$$


Given the bounded poset $\hat{Q}$, one can define $\hat{O}(Q)$ as the set of all functions $g$ such that

$$
\begin{aligned}
& g(\hat{0})=0, \\
& g(\hat{1})=1,
\end{aligned}
$$

and

$$
g(x) \leq g(y) \quad \text { if } y \text { covers } x \text { in } \hat{Q} .
$$

Then the bijective linear map $\rho: \hat{O}(Q) \rightarrow O(Q)$ given by restriction to $Q$ defines a combinatorial equivalence of polytopes. Stanley was able to derive the entire facial structure of $\hat{O}(Q)$ [32, Section 1]. In particular, the number of facets of $O(Q)$ is equal to the number of cover relations in $\hat{Q}$, and the number of vertices of $O(Q)$ is given by:

$$
\mid\{I \subset Q \mid \text { if } x \in I \text { and } y \geq x \text { then } y \in I\} \mid .
$$

Theorem 5.6 [32, Section 4]. Let $Q$ be a finite poset with $|Q|=d$. Then:

(i) $L_{O(Q)}(k)=\left|k O(Q) \cap \mathbb{Z}^{d}\right|=\Omega(Q, k+1)$ for each $k \in \mathbb{Z}$;

(ii) $\operatorname{vol}(O(Q))=e(Q) / d$ !.

Corollary 5.7. Let $Q$ be a finite poset with order polytope $P:=O(Q)$. Then

$$
\left|(k P)^{\circ} \cap \mathbb{Z}^{d}\right|=\bar{\Omega}(Q, k-1) \quad \text { for all } k \in \mathbb{Z}_{>0} .
$$

Proof. This is immediate from Theorems 2.3, 5.4(i), and 5.6(i):

$$
\begin{aligned}
L_{P^{\circ}}(k) & =(-1)^{d} L_{P}(-k) \\
& =(-1)^{d} \Omega(Q, 1-k) \\
& =\bar{\Omega}(Q, k-1) \quad \text { for any } k \in \mathbb{Z}_{\geq 0} .
\end{aligned}
$$

Remark 5.8. Suppose that $Q$ is graded of rank $r$. Stanley [33, Corollary 4.5.17] showed that

$$
\Omega(Q,-1)=\Omega(Q,-2)=\cdots=\Omega(Q,-r)=0
$$

and that

$$
\Omega(Q,-r-1)=(-1)^{d} .
$$

From Corollary 5.7 we see that $(r+2) O(Q)$ is the smallest integral dilation of $O(Q)$ with an interior lattice point; in fact $(r+2) O(Q)$ contains a unique interior lattice point. Proposition 5.9. Let $Q$ be a poset with $|Q|=d$. Let $P:=O(Q)$ be the order polytope of $Q$. Then the boundary volume of $P$ is

$$
\operatorname{vol}(\partial P)=\frac{(3-d) e(Q)+2 e_{d-1}(Q)}{(d-1) !} .
$$

If in addition $Q$ is a graded poset of rank $r$ then the boundary volume of $P$ is

$$
\operatorname{vol}(\partial P)=\frac{(r+2) e(Q)}{(d-1) !} .
$$


Proof. Since $L_{P}(k)=\Omega(Q, k+1)$ for each $k \in \mathbb{Z}$ by Theorem 5.6(i), if we express $\Omega(Q, n)$ as

$$
\Omega(Q, n)=\sum_{i=0}^{d} a_{i} n^{i}
$$

then

$$
L_{P}(n)=\Omega(Q, n+1)=a_{d}(n+1)^{d}+a_{d-1}(n+1)^{d-1}+\sum_{i=0}^{d-2} a_{i}(n+1)^{i} .
$$

If we express $L_{P}(n)$ in the form

$$
L_{P}(n)=\sum_{i=0}^{d} c_{i} n^{i}
$$

then $c_{d-1}=a_{d-1}+d a_{d}$.

Using Theorem 5.4(iv) and (v), we get that

$$
c_{d-1}=d \frac{e(Q)}{d !}+\frac{e_{d-1}(Q)}{(d-1) !}-\frac{\left(\begin{array}{l}
d \\
2
\end{array}\right) e(Q)}{d !}=\frac{e(Q)(3-d)+2 e_{d-1}(Q)}{2(d-1) !} .
$$

But it follows from Theorem 2.2(ii) that

$$
\frac{1}{2} \operatorname{vol}(\partial P)=c_{d-1}=\frac{e(Q)(3-d)+2 e_{d-1}(Q)}{2(d-1) !} .
$$

Combining these results gives

$$
\operatorname{vol}(\partial P)=\frac{(3-d) e(Q)+2 e_{d-1}(Q)}{(d-1) !} .
$$

When $Q$ is a graded poset, applying Theorem 5.4(vi) to the previous formula gives

$$
\operatorname{vol}(\partial P)=\frac{(r+2) e(Q)}{(d-1) !} .
$$

Lemma 5.10. Let $Q$ be a graded poset of rank $r$ with $|Q|=d$. Then $(r+2) O(Q)$ is a translate of a reflexive polytope.

Proof. Let $P:=(r+2) O(Q)$ be the $(r+2)$ th dilate of the order polytope $O(Q)$ of $Q$. It is enough to prove that $d \operatorname{vol}(P)=\operatorname{vol}(\partial P)$. But

$$
\begin{aligned}
d \operatorname{vol}(P) & =d \operatorname{vol}((r+2) O(Q)) \\
& =d(r+2)^{d} \operatorname{vol}(O(Q)) \\
& =d(r+2)^{d} \frac{e(Q)}{d !} \\
& =(r+2)^{d-1} \frac{(r+2) e(Q)}{(d-1) !} \\
& =(r+2)^{d-1} \operatorname{vol}(\partial O(Q)) \\
& =\operatorname{vol}(\partial P) .
\end{aligned}
$$


Since $(r+2) O(Q)$ is a (translate of a) reflexive polytope, we can reinterpret our results from Section 3 in terms of the order polytope.

Corollary 5.11 (See Corollary 3.6). Let $Q$ be a finite graded poset of rank $r$ with $|Q|=d$. Let $e(Q)$ denote the number of linear extensions of $Q$. Then

$$
(r+2)^{d} e(Q)=\sum_{m=0}^{n}(-1)^{n+m}\left(\left(\begin{array}{c}
d \\
n-m
\end{array}\right)+(-1)^{d-1}\left(\begin{array}{c}
d \\
n+m+1
\end{array}\right)\right) \Omega(Q, m(r+2)+1),
$$

where $n:=\lfloor d / 2\rfloor$.

Theorem 5.12 (See Theorem 3.8). Let $Q$ be a finite graded poset of rank $r$ with $|Q|=d$. Suppose that $d$ is odd. Then

$$
\sum_{m=0}^{N}(-1)^{N+m}\left(\begin{array}{c}
d+2 \\
N-m
\end{array}\right) \Omega(Q, m(r+2)+1)=0
$$

where $N:=\lceil d / 2\rceil$.

\section{The Birkhoff polytope}

Let $B(d)$ denote the Birkhoff polytope (or transportation polytope) of $d \times d$ doubly stochastic matrices in $\mathbb{R}^{d^{2}}$. That is, $B(d)$ is defined by

$$
x_{i, j} \geq 0, \quad \sum_{i=1}^{d} x_{i, j}=1, \quad \sum_{j=1}^{d} x_{i, j}=1 \quad \text { for all } 1 \leq i, j \leq d .
$$

Because of its rich combinatorial properties, the Birkhoff polytope has been intensively studied. In particular, methods for estimating and computing the volume and Ehrhart polynomial are of considerable interest (see [5, 9, 24]). The following theorem summarizes some of the key facts about $B(d)$.

TheOREM 6.1. Let $B(d)$ denote the polytope of $d \times d$ doubly stochastic matrices in $\mathbb{R}^{d^{2}}$. Let $H_{n}(r)$ denote the number of $n \times n$ magic squares with linear sums equal to $r$. Let $P_{n}(r)$ denote the number of $n \times n$ positive magic squares with linear sums equal to $r$, where 'positive' means that all entries of the matrix are positive. Then:

(i) $\operatorname{dim} B(d)=(d-1)^{2}$;

(ii) $L_{B(d)}(m)=H_{d}(m)$ for all $d \in \mathbb{Z}_{>0}$ and $m \in \mathbb{Z}_{\geq 0}$;

(iii) $L_{B(d)}(-d-t)=(-1)^{(d-1)^{2}} L_{B(d)}(t)$ for all $t \in \mathbb{Z}$;

(iv) the vertices of $B(d)$ are the permutation matrices;

(v) $\quad L_{B(d)^{\circ}}(m)=P_{d}(m)$ for all $d \in \mathbb{Z}_{>0}$ and $m \in \mathbb{Z}_{>0}$.

In fact-as the following two lemmas show-it is easy to see that the $d$ th dilation of the Birkhoff polytope contains precisely one interior lattice point, and that this dilation is a translate of a reflexive polytope. 
Lemma 6.2. Let $B(d)$ denote the polytope of $d \times d$ doubly stochastic matrices in $\mathbb{R}^{d^{2}}$. Then $\left|d B(d)^{\circ} \cap \mathbb{Z}^{d^{2}}\right|=1$.

Proof. Using Theorem 6.1(v),

$$
\left|d B(d)^{\circ} \cap \mathbb{Z}^{d^{2}}\right|=L_{B(d)^{\circ}}(d)=P_{d}(d) .
$$

But if $Q$ is a $d \times d$ positive magic square whose lines sum to $d$, then $Q$ must be the matrix with all entries equal to one. Hence $P_{d}(d)=1$.

Lemma 6.3. Let $P:=d B(d)-Q$ denote the translation of the dth dilate of the Birkhoff polytope by $Q$, where $Q$ is the matrix with all entries equal to one. Then $P$ is a reflexive polytope.

Proof. From Theorem 3.4(i) and (ii) it is enough to show that

$$
(-1)^{(d-1)^{2}} L_{d B(d)}(-m)=L_{d B(d)}(m-1)
$$

for all $m \in \mathbb{Z}_{>0}$. But setting $t=d(m-1)$ in Theorem 6.1(iii) gives:

$$
L_{d B(d)}(m)=L_{B(d)}(d m)=(-1)^{(d-1)^{2}} L_{B(d)}(d(m-1))=(-1)^{(d-1)^{2}} L_{d B(d)}(m-1) .
$$

We can now reinterpret our results in Section 3 in terms of the Birkhoff polytope. In particular, an explicit formula for the volume of the Birkhoff polytope is given in terms of the the first $\left\lfloor(d-1)^{2} / 2\right\rfloor$ dilations.

Corollary 6.4 (See Corollary 3.3). Let $B(d)$ denote the polytope of $d \times d$ doubly stochastic matrices in $\mathbb{R}^{d^{2}}$. Then

$$
\left((d-1)^{2}\right) ! d^{(d-1)^{2}} \operatorname{vol}(B(d)) \geq(d-1)^{2} H_{d}(d)-(d-1)^{2}+3 .
$$

Corollary 6.5 (See Corollary 3.6). Let $B(d)$ denote the polytope of $d \times d$ doubly stochastic matrices in $\mathbb{R}^{d^{2}}$. Then

$$
\operatorname{vol}(B(d))=\frac{1}{\left((d-1)^{2}\right) ! d^{(d-1)^{2}}} \sum_{m=0}^{n}(-1)^{n+m}\left(\left(\begin{array}{c}
(d-1)^{2} \\
n-m
\end{array}\right)+(-1)^{d}\left(\begin{array}{c}
(d-1)^{2} \\
n+m+1
\end{array}\right)\right) H_{d}(m d)
$$

where $n:=\left\lfloor(d-1)^{2} / 2\right\rfloor$.

Theorem 6.6 (See Theorem 3.8). Suppose that $d$ is even. Then

$$
\sum_{m=0}^{N}(-1)^{N+m}\left(\begin{array}{c}
d^{2}-2 d+3 \\
N-m
\end{array}\right) H_{d}(d m)=0
$$

where $N:=\left\lceil(d-1)^{2} / 2\right\rceil$. 


\section{Acknowledgements}

The authors would like to thank Josef Schicho and Benjamin Nill for many helpful remarks, and Don Taylor for alerting them to [34]. This work was completed whilst the second author was a guest at RicAm.

\section{References}

[1] V. V. Batyrev, 'On the classification of smooth projective toric varieties', Tohoku Math. J. 43(4) (1991), 569-585.

[2] V. V. Batyrev, 'Dual polyhedra and mirror symmetry for Calabi-Yau hypersurfaces in toric varieties', J. Algebraic Geom. 3(3) (1994), 493-535.

[3] V. V. Batyrev, 'On the classification of toric Fano 4-folds', J. Math. Sci. (New York) 94(1) (1999), 1021-1050.

[4] M. Beck, J. A. De Loera, M. Develin, J. Pfeifle and R. P. Stanley, 'Coefficients and roots of Ehrhart polynomials', in: Integer Points in Polyhedra-Geometry, Number Theory, Algebra, Optimization, Contemporary Mathematics, 374 (American Mathematical Society, Providence, RI, 2005), pp. 15-36.

[5] M. Beck and D. Pixton, 'The Ehrhart polynomial of the Birkhoff polytope', Discrete Comput. Geom. 30(4) (2003), 623-637.

[6] M. Beck and S. Robins, Computing the Continuous Discretely: Integer-Point Enumeration in Polyhedra, Undergraduate Texts in Mathematics (Springer, New York, 2007).

[7] M. Beck and F. Sottile, 'Irrational proofs for three theorems of Stanley', European J. Combin. 28(1) (2007), 403-409.

[8] D. Bremner and V. Klee, 'Inner diagonals of convex polytopes', J. Combin. Theory Ser. A 87(1) (1999), 175-197.

[9] E. R. Canfield and B. D. McKay, 'The asymptotic volume of the Birkhoff polytope', Online J. Anal. Comb. (4) (2009), Art. 2, 4.

[10] C. Casagrande, 'The number of vertices of a Fano polytope', Ann. Inst. Fourier (Grenoble) 56(1) (2006), 121-130.

[11] V. I. Danilov, 'The geometry of toric varieties', Uspekhi Mat. Nauk 33(2(200)) (1978), 85-134, 247.

[12] R. Diaz and S. Robins, 'The Ehrhart polynomial of a lattice polytope', Ann. of Math. (2) 145(3) (1997), 503-518.

[13] E. Ehrhart, 'Sur un problème de géométrie diophantienne linéaire. II. Systèmes diophantiens linéaires', J. Reine Angew. Math. 227 (1967), 25-49.

[14] M. H. J. Fiset and A. M. Kasprzyk, 'A note on palindromic $\delta$-vectors for certain rational polytopes', Electron. J. Combin. 15(N18).

[15] C. Haase and I. V. Melnikov, 'The reflexive dimension of a lattice polytope', Ann. Comb. 10 (2006), 211-217.

[16] T. Hibi, 'Ehrhart polynomials of convex polytopes, $h$-vectors of simplicial complexes, and nonsingular projective toric varieties', in: Discrete and Computational Geometry (New Brunswick, NJ, 1989/1990), DIMACS Series in Discrete Mathematics and Theoretical Computer Science, 6 (American Mathematical Society, Providence, RI, 1991), pp. 165-177.

[17] T. Hibi, 'A lower bound theorem for Ehrhart polynomials of convex polytopes', Adv. Math. 105(2) (1994), 162-165.

[18] A. M. Kasprzyk, 'Toric Fano varieties and convex polytopes', PhD Thesis, University of Bath, Available from http://hdl.handle.net/10247/458.

[19] V. Klee, 'A combinatorial analogue of Poincaré's duality theorem', Canad. J. Math. 16 (1964), 517-531.

[20] K. Kołodziejczyk, 'On the volume of lattice manifolds', Bull. Aust. Math. Soc. 61(2) (2000), 313-318. 
[21] I. G. Macdonald, 'The volume of a lattice polyhedron', Proc. Cambridge Philos. Soc. 59 (1963), 719-726.

[22] I. G. Macdonald, 'Polynomials associated with finite cell-complexes', J. Lond. Math. Soc. (2) 4 (1971), 181-192.

[23] M. Øbro, 'An algorithm for the classification of smooth Fano polytopes', arXiv:0704.0049v1 [math.CO], Classifications available from http://grdb.lboro.ac.uk/.

[24] I. Pak, 'Four questions on Birkhoff polytope', Ann. Comb. 4(1) (2000), 83-90.

[25] H. S. Park, 'The $f$-vectors of some toric Fano varieties', J. Appl. Math. Comput. 11(1-2) (2003), 437-444.

[26] G. A. Pick, 'Geometrisches zur Zahlenlehre', Sitzungber. Lotos 19 (1899), 311-319.

[27] J. E. Pommersheim, 'Toric varieties, lattice points and Dedekind sums', Math. Ann. 295(1) (1993), $1-24$.

[28] J. E. Reeve, 'On the volume of lattice polyhedra', Proc. Lond. Math. Soc. (3) 7 (1957), 378-395.

[29] R. P. Stanley, 'A chromatic-like polynomial for ordered sets', in: Proc. Second Chapel Hill Conf. on Combinatorial Mathematics and its Applications (Univ. North Carolina, Chapel Hill, N.C., 1970) (Univ. North Carolina, Chapel Hill, N.C, 1970), pp. 421-427.

[30] R. P. Stanley, 'Hilbert functions of graded algebras', Adv. Math. 28(1) (1978), 57-83.

[31] R. P. Stanley, 'Decompositions of rational convex polytopes', Ann. Discrete Math. 6 (1980), 333-342; Combinatorial Mathematics, Optimal Designs and their Applications (Proc. Sympos. Combin. Math. and Optimal Design, Colorado State Univ., Fort Collins, Colo., 1978).

[32] R. P. Stanley, 'Two poset polytopes', Discrete Comput. Geom. 1(1) (1986), 9-23.

[33] R. P. Stanley, Enumerative Combinatorics Vol. 1, Cambridge Studies in Advanced Mathematics, 49 (Cambridge University Press, Cambridge, 1997), with a foreword by Gian-Carlo Rota. Corrected reprint of the 1986 original.

[34] L. R. Turner, 'Inverse of the Vandermonde matrix with applications'. NASA Technical Note.

\section{GÁBOR HEGEDÜS,}

Johann Radon Institute for Computational and Applied Mathematics, Austrian Academy of Sciences, Altenbergerstraße 69, A-4040 Linz, Austria e-mail: gabor.hegedues@oeaw.ac.at

ALEXANDER M. KASPRZYK, Department of Mathematics, Imperial College London, London SW7 2AZ, United Kingdom e-mail: a.m.kasprzyk@imperial.ac.uk 